\title{
To Leverage the Innovation Capability by Co-creation of Human-Machine
}

\author{
Shuxian Chen ${ }^{1}$, Jiaona Xiang ${ }^{1}$, Zongqiang Ren $^{2 *}$ \\ ${ }^{1}$ School of Business, Wenzhou University, Zhejiang, China \\ ${ }^{2}$ Wenzhouness Economy Research Institute, Wenzhou University, Zhejiang, China \\ *Corresponding Author.
}

\begin{abstract}
:
Although a societal consensus has been reached about the partnership between human beings and smart machines, limited research has been carried out to consider advances in its combination pattern, function mechanism, and developing creativity. Based on occurred disruptive changes form theory to practice, a new paradigm of co-creation by synergistic human-machine is proposed, which refers to that interaction of human-machine learning in a holistic system increase complementarity of abilities and integration of wisdom to realize an augmented synergy made up of hybrid intelligences. This augmented intelligence can effectively account for shifts in business logic and the productive solutions to the multiple complex problems during smart manufacturing. It has been discovered that the positive consequences of synergistic human-machine co-creation include not only sustainable labor patterns, but the ability to overcome multiple complexities and steadily increase business value. All this is further elaborated in the Baizhentang Foods case. This paper discusses the main prospects of the theoretical developments presented here for future research on organizational behavior and a synergistic human-machine structure.
\end{abstract}

Keywords: Synergistic human-machine, Co-creation, Smart manufacturing, Paradigm.

\section{INTRODUCTION}

The New Industrial Revolution is characterized by a new wave of technological and industrial revolution associated with the combination of digitization, networking, intelligence, and advanced manufacturing and an accelerated reconfiguration of the economic landscape [1]. Smart manufacturing has become a significant leverage point to upgrade the industrial chain and overcome economic stagnation for many countries. Although the complete transition to Industry 4.0 may still take 20 years, it is conspicuous that the idea of smart machines' 
mimicking human cognition nourishes the most disruptive empowerment [2-4]. The role of the machine has shifted from a collaborative tool for people to attain efficient production to an enabler of joint innovation promotion with human beings, supported by recent advances in computational power, the exponential increase in data availability, and new machine learning techniques [5]. Developing a sound theory that situates the ongoing collaboration mode between human and machine is important because such collaboration has been creeping into the whole process of organizational operation and management. In this new era, advances in pattern combinations, function mechanisms, relation modes, and hierarchy development will become the essential indicators of productivity promotion [6-8]. It goes without saying that creative contributions to this new mode of working have developed in greater scope and depth than ever.

Today, collaborative human-machine teamwork has become a widespread consensus in all walks of life [9-15]. Nonetheless, many human managers lack trust in intelligent machines and are more willing to gain a sense of freedom from cooperation by prioritizing domination [10, 16]. Available studies have mostly emphasized how to achieve shared control of a human-machine collaboration assisted by an interface and have paid less attention to exploring collaborative human-machine functions from the perspectives of humanity and management. Briefly, these anthropocentric studies do not allow enough maneuvering room for smart machines to account for how collaborative behaviors between humans and machines achieve synergies like swarm intelligence $[17,18]$.

Hence, there is a need to develop more explicit theories of the patterns in which humans collaborate with machines as well as the co-creative mechanism that highlights the process and its consequences, specifically with respect to smart manufacturing. For this purpose, the concept of paradigm shift was used in this work to develop a theoretical framework of synergistic human-machine co-creation. The following sections present three critical backgrounds that highlight the importance of considering new technological trajectories and the emerging dominant design. Next, the study drew on the dynamic development between productivity and production relations, including production factors, working modes, innovative agents, and value systems. The next step was to develop a framework for human-machine synergy as a basis for co-creation and subsequently to propose the concept of synergistic human-machine co-innovation. Building on a case at Baizhentang Foods in China, this study shows how food enterprises have developed smart manufacturing through synergistic human-machine co-innovation. Finally, a discussion of the perspectives of the proposed paradigm for future research on organizational behavior and structural relationship concludes the paper. 


\section{BACKGROUND}

\subsection{Smart Manufacturing Requisitions toward Human-Machine}

Smart manufacturing originated from intelligence research and was first defined in Manufacturing Intelligence [19]. It emphasized that a smart machine could independently produce small quantities under the condition of integrated knowledge engineering, sensors, and other technologies. As these emerging technologies have rapidly developed, smart manufacturing has further developed from automation and digitization to deep intelligence. This has entirely overturned the mechanical manufacturing tradition, enabling industrial giants to achieve strong and sustainable productivity as well as the wisdom of lean management. Each manufacturing plant provides not only perfect communication and cooperation between workers, but also real-time and barrier-free interaction between workers and machines to share information [20]. The role of the intelligent system consisting of human experts and smart machines is much more important in manipulating multiple assembly lines during the manufacturing process. Specifically, the actions of this system such as self-learning, analyzing, and judging based on information access contribute to resolving the uncertainties of the manufacturing system, including diversity of customer demand, small-scale orders, and supply chain changes [20]. Obviously, human-machine collaboration will help our society discover more opportunities in an intelligent and complex environment with these disruptive enablers, which may imply the growth of a novel innovation paradigm [21].

\subsection{Transformation of the Collaboration between Human and Machine}

Human beings are born with unique wisdom in behavior, relationships, and psychology [22], which enables them to alter their thoughts by an act of will and to bring much greater flexibility and creativity to dealing with cases [23, 24]. Therefore, machines used to mimic the learning process of biological intelligence, especially tracking humans' long short-term memory, recurrent neural networks, and multitask learning [25]. Gradually, they acquired more abilities, from simple mechanization to computerized cognition and even semi-automatic or fully automatic emotion. Eventually, machine intelligence may catch up with or even transcend human wisdom in some fields with the progress of machine learning and its self-iteration. For example, Microsoft Image Composite Editor, an intelligent writing machine, has published two poetry collections. From the viewpoint of epistemology, the machine has become a new agent [26] that can cooperate with human beings at work [27-29] and that can liberate people from trivial and repetitive tasks to jointly improve the efficiency of the individual and the 
organization. Moreover, using the capabilities of Big Data filtering and real-time processing, machines can participate in decision-making with human beings, which would shift the previous logic that relied upon traditional tools and experiences. In the past decade, cooperation and mutual benefit between human and machine have become a hot topic in the field of artificial intelligence and business innovation [30]. An increasing number of studies have proved that it is far from enough to solve specific problems only by human or only by machine independently [26, 31]. Work in the future will rely more on the cooperative human-machine model [15, 32], which has increasingly become the core force of societal creation.

\subsection{Paradigm Shift of Management Innovation}

Each previous Industrial Revolution brought creative disruption to the contemporary industrial system and production mode and then gave birth to emerging industries based on major technological breakthroughs. In other words, the techno-economic paradigm has repeatedly undergone a revolution through reconfiguring and multiplying essential production factors to break the limits of growth from the perspective of evolutionary economics [33-37]. Taking four generations of industrial revolutions as an example, shifts in management logic occurred through substitutions among steam, steel, electricity, and computing whereby the old techno-economic paradigm is marginalized and the new one gradually becomes dominant [38]. Regarding the four ages, [39] teased out a sequence of management modes running from line-and-staff to scientific management and strategy-and-structure to business process mode. However, R\&D mechanisms and technology trajectories ordinarily vary between different technology clusters or different innovative situations [40]. Since the 1950s, there have been five generations of technological innovation. The trajectories are respectively technology-driven, demand-pull, market-technology interaction, integrated innovation, and systematic integration network [41]. After Rothwell, Nobelius [41, 42] proposed six R\&D management models to describe advances based on the laboratory, business unit, project, cross-department, cross-border alliances, and loose network. Recently, Chinese researchers have summarized different innovation paradigms that have emerged in diverse nations in the past 30 years, such as user innovation, open innovation, simple innovation, and responsible innovation [43]. These theories all emphasized arousing humans' creative efforts by efficiently optimizing resource allocation with new technologies, each of which was also highly consistent with the required conditions in different periods, and which established the fundamental role of the techno-economic paradigm in leading industrial development [36, 37].

Nevertheless, as mentioned above, a consensus has appeared in all walks of life that smart machines and humans must become colleagues [44, 45]. A new age of human-machine 
co-creation bringing the scientific research of human understanding of the world into the fourth paradigm is coming, which may make existing theories obsolete because of outdated prerequisites [31, 46]. This will be not only a technological revolution, but also a renewal of thinking and cognition. Hence, researchers have appealed for a new management revolution to accelerate the formation of the dominant design paradigm that accompanies every new technological revolution [34, 47]. It is urgent to re-examine the changes in productivity and production relations brought about by new human-machine combinations and to explore the innovation models that can adapt to this situation from the standpoint of paradigm shift [48].

\section{UPGRADING CO-CREATION OF HUMAN-MACHINE SYNERGY}

As Kurzwell predicted in The Singularity is Near, intelligence development in the next generation will be up to the combination of humans and machines [49]. Holistically embedding humans' wisdom and machines' intelligence will contribute to the growth of this paradigm in various domains [50], especially the positive effects on societal innovation and business behavior $[5,28]$. The synergistic human-machine innovation process model leads to compatible development of productivity and production relations, including production factors, working modes, innovative agents, and value systems.

\subsection{Production Factors: From Assets to Intelligence}

Machines are no longer used as assets, but as smart agents because they have acquired spontaneous representation, reasoning, learning, and adaptation by mimicking biological intelligence. For instance, machines can translate data into wisdom by step-through coding logic and rules with integrated equations [25, 51]. Therefore, it is likely that machine intelligence will be devoted to the production value stream to move the production mode from efficiency to innovation $[29,52]$. In this scenario, machines will work closely with humans, which is made possible by digitization, dynamics, and the timeliness of experience and knowledge. What has happened nowadays is that data and machines have become new production factors and a new labor force [29]. This breaks down the restrictions of traditional factors like the limited supply of human labor, capital, and land that have impeded large-scale sustainable development.

\subsection{Work Mode: From Separation to Coupling}

In earlier times, machines working in a separate or partially shared workplace completely obeyed humans' unilateral commands. This was basically a coexistence working mode without 
any coupling process. However, the behaviors of humans and machines have become much more highly interactive with the maturity of artificial intelligence, especially now that the learning trajectories of intelligence are no longer separated. For example, machines can analyze situations, recognize images, and use deep learning to approach human thinking [53, 54], which enables them to perform cooperative work involving close interaction. More importantly, the two can even achieve goal sharing that is not only limited to the co-workspace [10]. The work mode will develop by leaps and bounds from separation to coexistence, from coexistence to coordination, and from coordination to collaboration [28, 55].

\subsection{Innovative Agents: Enter a New Collaborative Era of Dualistic Agents}

Human beings are endowed with skills in improvisation, agile response, evaluation, and leadership [28], whereas artificially intelligent beings are expert in speed, accuracy, repetition, prediction, and measurability to an extent that human intelligence cannot match. This enables machines to play an essential role in identifying, uncovering, processing, and utilizing Big Data to diagnose potential disruption of the value chain [30]. When integrating machine learning with human knowledge so that they can evolve together, the domains of work performed by machines will gradually expand to a higher level, and human beings will become more absorbed in the interests and expertise of personal creativity, which is more likely to stimulate and efficiently realize intelligent innovation $[28,29,56]$. For example, a heterogeneous human-machine agent may possess the ability of foresight, which entirely reconstructs the societal production and distribution mode based on incremental innovation to create production value. The relationship between humans and machines has developed from servitude to partnership [57], and more and more people are calling on us to regard smart machines as new colleagues [45]. Societal innovation has entered a new collaborative era of dualistic agents. 


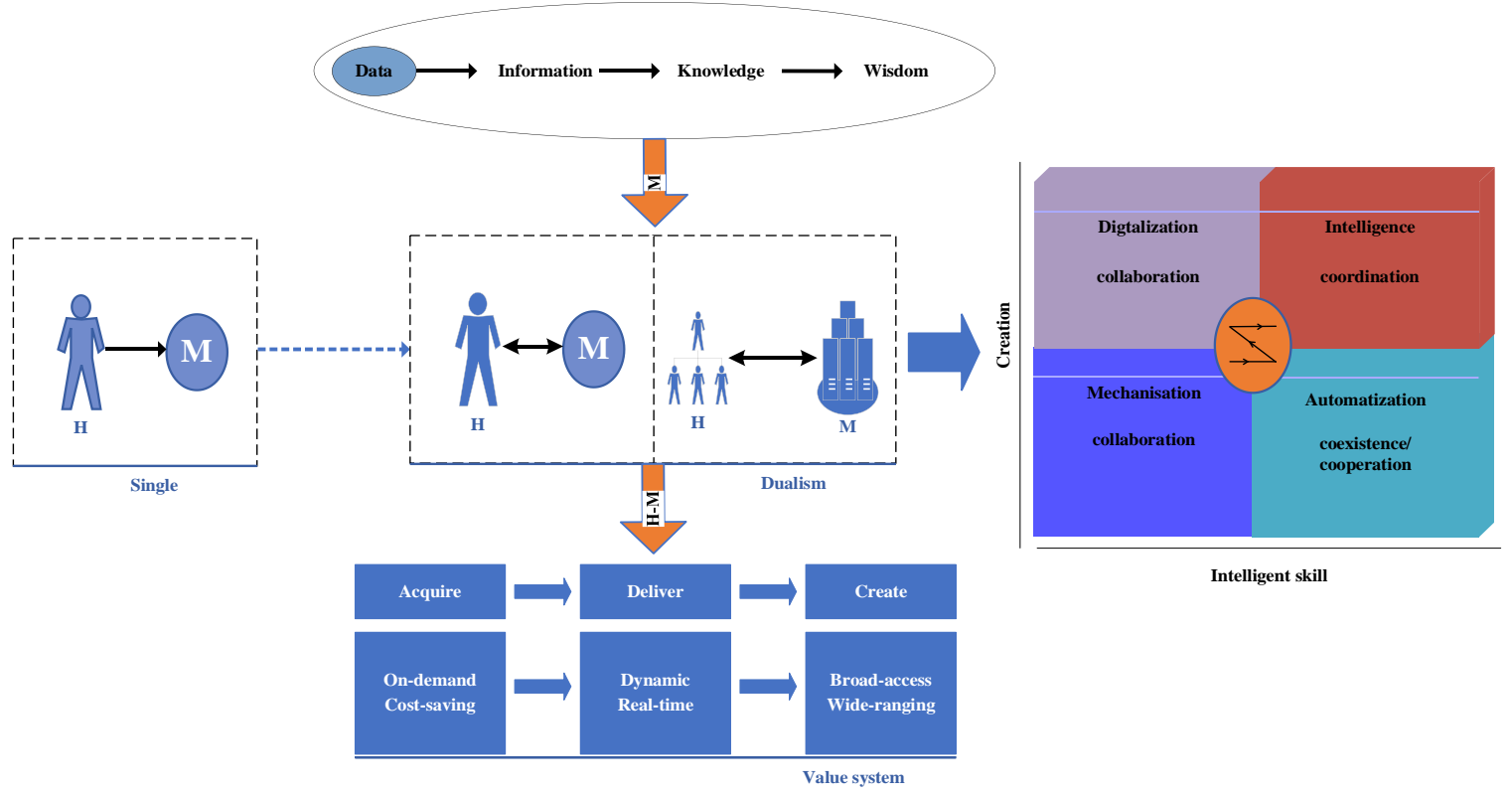

Fig. 1: shifts in productivity and production relations

3.4 Value System: Trending to the Societal, Ubiquitous, On-Demand Logic of Creation

A synergistic human-machine system can create scientific insights driven by data as well as rooted in humans' societal cognition. Therefore, the decisions made by the holistic organism may well reduce the bias caused by overfitting or empiricism and become widely used to solve multiple manufacturing problems under the conditions of digital technology with strong connectivity, cognitive ability, and imperceptibility [30]. This gradually leads to a dynamic societal, on-demand, and wide-ranging value system. For example, situational judgments by synergistic human-machine entities can help enterprises dynamically coordinate the allocation of production factors to achieve incremental value through lean production. Evidently, the synergistic human-machine system is now rejiggering the value system of innovation subjects from three salient aspects through full integration of the wisdom consensus of machines and humans, including value acquisition, value delivery, and value creation, which manifests new characteristics as shown in Fig. 1.

\section{CONNOTATIONS OF SYNERGISTIC HUMAN-MACHINE CO-CREATION}

A collaborative human-machine system can be simply regarded as a computational 
reasoning system composed of humans and machines [26]. However, co-innovation by a synergistic human-machine system emphasizes both the process and the consequence. In this paper, the connotation is that humans and smart machines achieve collaboration in work and integrated advantages in wisdom through the system. This process promotes frequent learning interactions and then finds the best value function or strategy by better knowledge integration with efficient reuse. This is a process of forming hybrid intelligence and generating augmented intelligence. Fig. 2 shows the framework of co-innovation by the synergistic human-machine system, which can be divided into four steps:

1) Humans and machines obtain raw data and events by real-time sensing through IoT and then extract the information they desire through the knowledge network;

2) Machines handle objective information, whereas humans process value-oriented information, including emergencies, and in turn each one upgrades the other's learning [58];

3) Humans and machines will make decisions in consensus using complementary computing and integrated reasoning to flexibly control labor or other factors, which formulates a smartly holistic controlled closed loop that can perceive, recognize and respond quickly and accurately;

4) By extension, co-innovation by a synergistic human-machine system can be much broader and more open if it links with the outside world. 


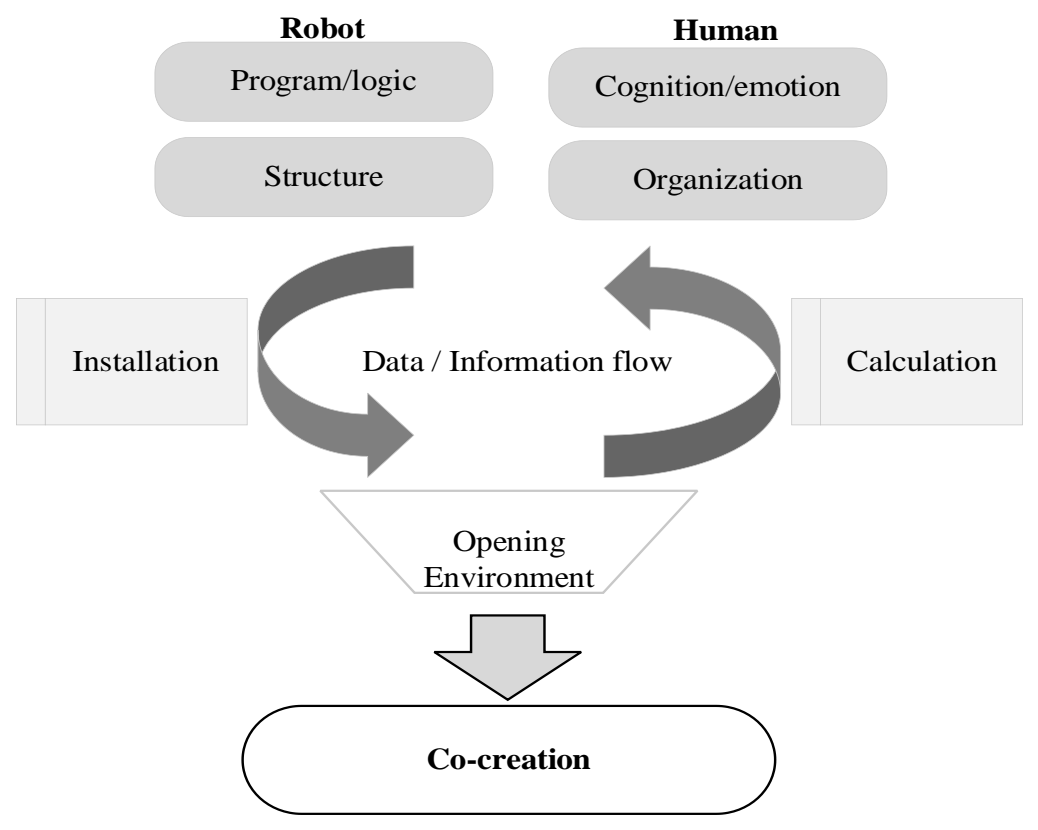

Fig. 2: framework of co-innovation by a synergistic human-machine system

Unlike an intelligent decision support system, co-innovation by a synergistic human-machine entity is a closed-loop bidirectional system that contains organisms and smart devices. They can seamlessly interact and integrate their wisdom to generate synergy to upgrade manufacturing, rather than being a dichotomous system of human helping machine or machine helping human [59]. First, the access to interaction has evolved from mechanical commands to multi-channel and multi-mode natural interaction. Second, the symbiotic relationship of the two components is not only on a physical level, but more to jointly understand and innovate the world in an unprecedented way, which strongly highlights goal orientation. Therefore, machine intelligence in use can be linked to corresponding societal logic by integrating human wisdom, which in turn is tested by objective algorithms and models, to induce the promotion of smart machines' ability and the enhanced creativity of humans.

\section{ILLUSTRATION OF THE PARADIGM BASED ON A CASE}

To realize production standardization and hygiene and guarantee product safety, the food industry has always adhered to high human-labor employment. However, even if the selecting, weighing, bagging, sealing, transporting and other processes are completed accurately and in compliance with requirements, traditional manufacturing still cannot avoid secondary pollution 
of food. This is because humans cannot naturally work under relatively low oxygen, but this is a special environmental requirement for food processing. However, the characteristics of a synergistic human-machine system are perfectly suited for this solution. With hybrid and augmented intelligence emerging from co-creation under the core model of synergistic integration between human and machine displayed in Fig.3, the food industry can achieve flexible production, which can quickly respond to expanding consumption.

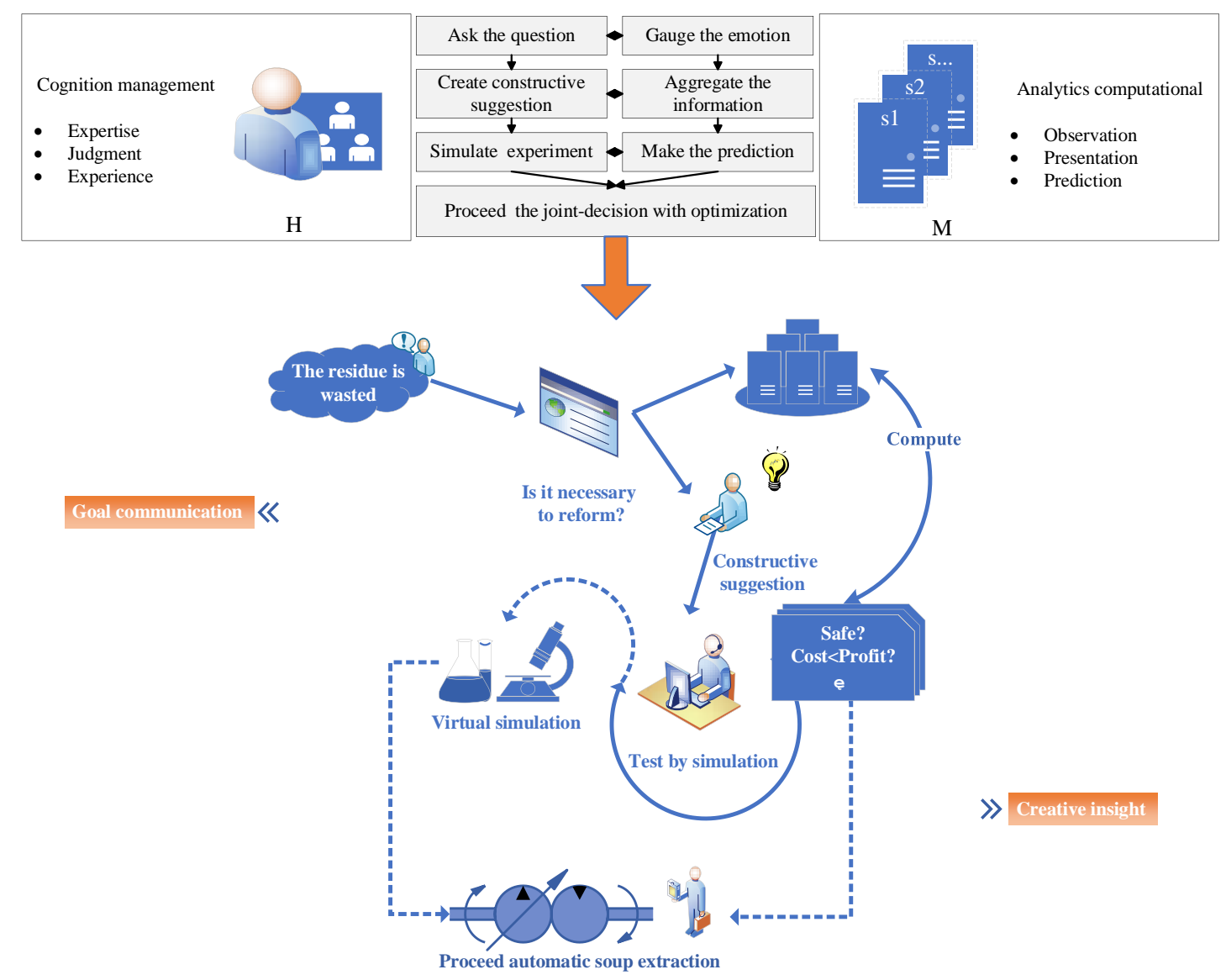

Fig. 3: the model of Synergistic work between human and machine

A practical case that focused on the positive impacts of applying synergistic human-machine co-creation to its production and management is the Baizhentang Foods enterprise. First, the use of smart devices, such as an automatic loading system with manipulators and a robot palletizing system, realizes unmanned production in the best environment for the eight major manufacturing segments of soup extraction, central control, 
cleaning, palletizing, and quick freezing. This further induces workers to become more immersed in the creative work of testing and developing food products to accelerate product innovation. Moreover, the production line can also be flexibly arranged, again taking advantage of integration with intelligent autonomy from both humans and smart machines to move from uncertainty, diversity, and complexity to agility, focus, and convergence. This will provide the enterprise with possibilities for broadening access to markets as well as widening the range of action to obtain twice the productivity with half the effort. Second, the enterprise uses a central control system to interconnect all the smart devices to achieve data visualization of the whole supply chain, guaranteeing feedback about any problems occurring during the whole management process. On the one hand, this structure enables machines to allocate production factors in an orderly manner to maximize benefits. Moreover, machines can help humans quickly distinguish potential threats to the business, such as slow delivery or slow scale-up, explore their reasoning with Big Data analysis, and even create a niche by drawing from more nuanced information. On the other hand, enhancing traceability and control by management enables human workers to aim at essential targets or curves. For example, by analyzing the time that franchisees, who are nearest to the market, take to process a dish for customers and comparing that with other competitors, new ideas for product development may be cultivated. Now this enterprise has obtained stronger market competitiveness in an intensive and proficient co-creation mode by synergistic human-machine systems and has become popular for its "Little Golden Bowl" of soup, which has quickly propelled it to the front of smart manufacturing.

\section{CONCLUDING RECOMMENDATIONS}

The maturity and breakthrough of information technology has led to an increase in the centrality of machines as smart agents, even though we are still far from the scientific singularity. When Elon Musk, who has created the Neuralink brain-machine interface chip, is attempting to realize hybrid human-machine intelligence from science fiction, it is obvious that co-creation by synergistic human-machine entities appears to be an irresistible force and that such symbiosis will become a new form of ecological organization. In this paper, regarding smart manufacturing and human-machine collaboration, a new model of collaborative creation by synergistic human-machine entities is presented, based on a techno-economic paradigm shift. The paper then proceeds to discuss the disruptive changes resulting from this model, including production factors, working modes, innovative agents, and value systems. This underlines the urgency of formulating new rules to promote the understanding and practice of human-machine synergy for innovation. Otherwise, the superiority of manufacturing will be compromised due to the lack of intrinsic intelligence that cannot cope with the deep shock of the New Industrial Revolution. This paper explicitly conceptualizes synergistic human-machine 
collaborative innovation as a novel innovation paradigm, referring to the fact that frequent human-machine learning interactions will enable increased complementarity of abilities and increased integration of wisdom to realize an synergy. This system will consist of a hybrid intelligence and establish augmented intelligence for the benefit of sustainable labor, overcoming multiple complexities, and steadily growing business value (seen in Fig.4). The application of these functions in manufacturing has been illustrated by the case of Baizhentang Foods. In conclusion, smart manufacturing in the future will become increasingly the province of co-creation by synergistic human-machine entities to generate positive consequences, such as exploring small-but-excellent rules to accurately predict the future through holistic knowledge generation and then to add new achievements in each transformation period.

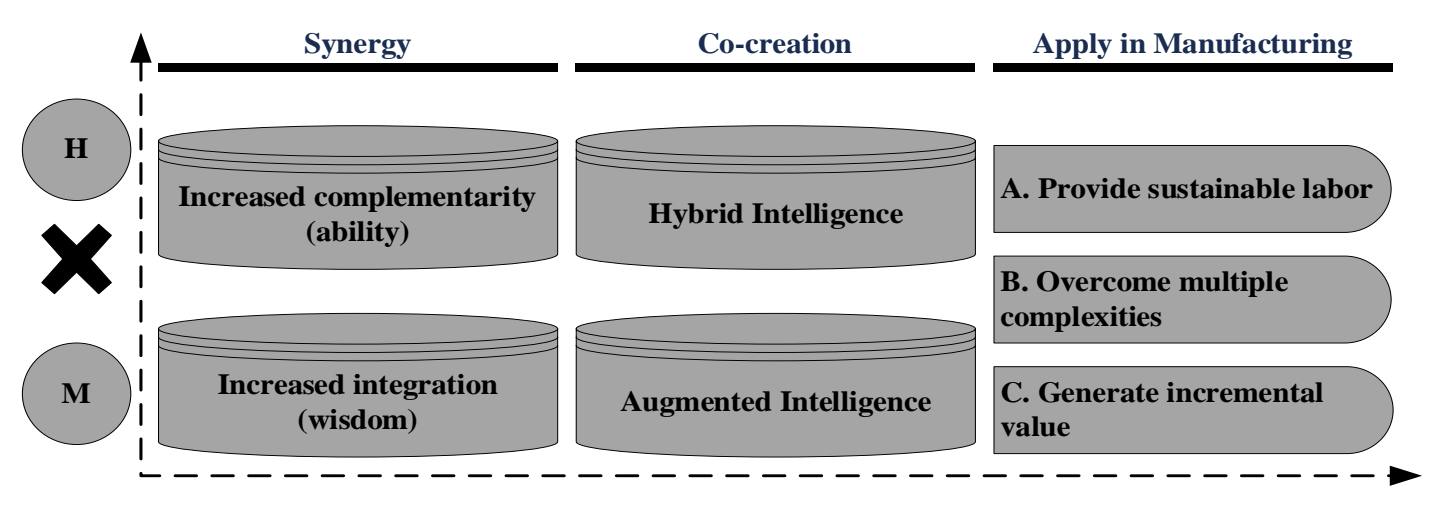

Fig. 4: synergistic human-machine co-creation toward smart manufacturing

Because the theoretical basis of co-innovation by synergistic human-machine systems is still in its initial stage, several prospects for its effect on organizational behavior and structural relationship are proposed in this paper. First, the design of synergistic human-machine co-innovation systems involves many aspects, particularly regarding actions, morality, ethics, authority, and hierarchy between humans and machines. Accordingly, it is worthwhile to further explore the interactions among roles and positions from the perspective of logic and ethics. As mentioned earlier, smart machines are no longer tools attached to human beings, but they have established a symbiotic and dualistic relationship with humans. At this point, it is essential to consider the position and interaction of the new community of human-machine systems in different situations, transcending anthropocentrism and metaphysical materialism [52]. However, after considering these factors, it remains to be answered how the network and organization of synergistic human-machine systems will evolve to function well in manufacturing processes such as $R \& D$, production, and sales. For instance, it will be necessary 
to explore an appropriate structure for human-machine systems to collaborate and then maximize the synergistic effect among technologies to generate powerful business values. Third, research on innovative mechanisms for synergistic human-machine entities is important to perfect the new paradigm, including the mechanisms of co-learning and knowledge creation by human-machine systems and the processes and modes of their steadily improving creativity. The former may aim to reveal knowledge taciturnity among machines and the knowledge-creation dualism between humans and machines. The latter includes the impact of smart technologies on human creativity, approaches to achieve hybrid intelligence by synergistic human-machine systems, and how to strengthen human creativity along with the interaction of humans and machines.

\section{ACKNOWLEDGMENTS}

This work was supported by National Office for Philosophy and Social Sciences (17BGL044) for the research on the promotion mechanism and path of innovation capability of traditional enterprises for smart manufacturing. We thank International Science Editing (http://www.internationalscienceediting.com) for editing this manuscript.

\section{REFERENCES}

[1] Lu, Y. (2017). Industry 4.0: A survey on technologies, applications and open research issues. Journal of Industrial Information Integration,6:1-10. doi:10.1016/j.jii.2017.04.005.

[2] Brynjolfsson, E., \& McAfee, A. (2017). The business of artificial intelligence. Harvard Business Review, 1-20.

[3] Raisch, S., \& Krakowski, S. (2020). Artificial intelligence and management: The automation-augmentation paradox. The Academy of Management Review, 1-48.

[4] Russell, S., \& Norvig, P. (1995). Artificial intelligence: a modern approach, Applied Mechanics \& Materials, 263(5), 2829-2833.

[5] Demir, K. A., Döven, G., \& Sezen, B. (2019). Industry 5.0 and human-robot co-working. Procedia Computer Science, 158, 688-695.

[6] Bostrom, N. (2016). Super intelligence. Computer Science.

[7] Zhao, Y. (2019). Human-machine relationship and information communication reform. Modern Communication (Journal of Communication University of China), 41(6), 150-154 (in Chinese).

[8] Tamura, Y., Egawa, M., Yano, S., Maeda, T., Asama, H. (2013). Activeness Improves Cognitive Performance in Human-Machine Interaction. Journal of Advanced Computational Intelligence and Intelligent Informatics (3), doi:10.20965/jaciii.2013.p0425.

[9] Bailey, D. E., \& Barley, S. R. (2019). Beyond design and use: How scholars should study intelligent technologies. Information and Organization, 30(2), 100286. 
[10] Bagdasarov, Z., Martin, A. A., \& Buckley, M. R. (2020). Working with robots: Organizational considerations. Organizational Dynamics, 49(2), 1-8.

[11] Davenport, T. H., \& Kirby, J. (2016). Just how smart are smart machines? MIT Sloan Management Review (57), 21-25.

[12] David, D. C. (2020). Leadership by Algorithm. Hampshire: Harriman House Ltd., 1-212.

[13] Grnsund, T. R., \& Aanestad, M. (2020). Augmenting the algorithm: Emerging human-in-the-loop work configurations. Journal of Strategic Information Systems, 29(2), 101614.

[14] Malone, T. W. (2018). How human-computer 'superminds' are redefining the future of work. MIT Sloan Management Review, 59(4), 34-41.

[15] Seeber, I., Bittner, E., Briggs, R. O., et al. (2018). Machines as teammates: A collaboration research agenda. 51st Hawaii International Conference on System Sciences, 420-429.

[16] Haesevoets, T., Cremer, D. D., Dierckx, K., \& Van Hiel, A. (2021). Human-machine collaboration in managerial decision making. Computers in Human Behavior, 119, 106730.

[17] Adadi, A., \& Berrada, M. (2018). Peeking inside the black-box: A survey on Explainable Artificial Intelligence (XAI). IEEE Access, 6, 52138-52160.

[18] Gillath, O., Ai, T., Branicky, M. S., Keshmiri, S., Davison, R. B., \& Spaulding, R. (2021). Attachment and trust in artificial intelligence. Computers in Human Behavior, 115, 106607.

[19] Wright, P. K., \& Bourne, D. A. (1988). Manufacturing Intelligence. Addison-Wesley.

[20] Chen, M., Liang N. M., et al. (2016). The Road to Intelligent Manufacturing: Digital Factory. Machinery Industry Press: Beijing, China (in Chinese).

[21] Kang, H. S. Lee, J. Y., Choi, S. S., et al. (2016). Smart manufacturing: Past research, present findings, and future directions. International Journal of Precision Engineering and Manufacturing-Green Technology,3(1):111-128.

[22] Jennings, N.R., Sycara, K., Wooldridge, M. (1998). A roadmap of agent research and development. Autonomous Agents and Multi-Agent Systems, 1(1), 7-38.

[23] Hooey, B.L., Gore, B.F., Wickens, C.D., Scott-Nash, S., Gov. (2011). Modeling Pilot Situation Awareness. Human Modelling in Assisted Transportation.

[24] Wickens, C., Mccarley, J., Thomas, L. (2003). Attention-Situation Awareness (ASA) Model. NASA Aviation Safety Program Conference on Human Performance Modeling of Approach and Landing with Augmented Displays.

[25] Deng, C., Ji, X., Rainey, C., Zhang, J., \& Lu, W. (2020). Integrating machine learning with human knowledge. iScience, 23(11), 101656.

[26] Luger, G.F., Pearson. (2002). Artificial Intelligence: Structures and Strategies for Complex Problem Solving: International Edition. Addison-Wesley Publishing Company.

[27] Pacaux-Lemoine, M.-P., Trentesaux, D., Rey, G. Z., \& Millot, P. (2017). Designing intelligent manufacturing systems through human-machine cooperation principles: A human-centered approach. Computers \& Industrial Engineering, 111, 581-595.

[28] Paul, R., Daugherty, H., Wilson, J. (2018). Human + Machine: Reimagining Work in the Age of AI. Harvard Business Review Press: Boston, 1-264. 
[29] Xie, X.F. (2019). Intelligent innovation of cultural and creative industries in the age of artificial intelligence: Paradigms and boundaries. Tongji University Journal Social Science Section, 30(1), 42-51(in Chinese).

[30] Canhoto, A.I., \& Clear, F. (2020). Artificial intelligence and machine learning as business tools: A framework for diagnosing value destruction potential. Business Horizons, 63, 183-193.

[31] Hey, T. (2010). The next scientific revolution. Harvard Business Review, 1, 1-14.

[32] Owana, N. (2018). Hyundai exoskeleton aims to cut workers' strains, will be tested in factories. https://techxplore.com/.

[33] Carlota, P. (2010). Technological revolutions and techno-economic paradigms. Cambridge Journal of Economics, 34(1), 185-202.

[34] Dosi, G., Freeman, C., Nelson, R., \& Soete, L. (1988). Technical Change and Economic Theory. London: Pinter, 1-646.

[35] Perez, C. (2003). Technological Revolutions and Financial Capital: The Dynamics of Bubbles and Golden Ages. England: Edward Elgar Publications, 1-224.

[36] Watanabe, C., Matsumoto, K., Griffy-Brown, C. (2001). Development and diffusion trajectory of innovative products in the light of institutional maturity-a comparative empirical analysis of the laser beam printer and optical cards. Technovation, 21(10), 637-647.

[37] Castellacci, F. (2007). Technological paradigms, regimes and trajectories: manufacturing and service industries in a new taxonomy of sectoral patterns of innovation. Research Policy, 37(6-7), 978-994.

[38] Dosi, G. (1982). Technological paradigms and technological trajectories: A suggested interpretation of the determinants and directions of technical change. Research Policy, 11(3), 147-162.

[39] Bodrozic, Z., \& Adler, P. S. (2018). The evolution of management models: A neo-Schumpeterian theory. Administrative Science Quarterly, 63(1), 85-129.

[40] Parayil, G. (2003). Mapping technological trajectories of the green revolution and the gene revolution from modernization to globalization. Research Policy, 32(6), 971-990.

[41] Rothwell, R. (1994). Towards the fifth-generation innovation process. International Marketing Review, 11(7), 7-31.

[42] Nobelius, D. (2004). Linking product development to applied research: Transfer experiences from an automotive company. Technovation, 24(4), 321-334.

[43] Chen, J., Yin, X. M., Mei, L. (2017). Holistic innovation: An emerging innovation paradigm based on Eastern wisdom. Technology Economics, 36(12): 1-10+29(in Chinese).

[44] Frick, W. (2015). When your boss wears metal pants. Harvard Business Review, 6, 84-89.

[45] Kolbjornsrud, V., Amico, R., \& Thomas, R. J. (2016). How artificial intelligence will redefine management. Harvard Business Review, 11(2), 2-6.

[46] Burkus, D. \& Zhao, J. O. (2017). The new management revolution: How the knowledge economy is reshaping organizations and management, how leading organizations are upending business as usual. CITIC Publishing Group Corporation.

[47] Chen, J. G. (2013). A hundred years of management and Chinese management innovation and development. Economic Management, 35(03),195-199(in Chinese).

[48] Kuhn, K. S. (1962). The Structure of Scientific Revolution. America Chicago: University of Chicago Press, 1-212. 
[49] Kurzwell, R. (2011). The Singularity is Near. Beijing: China Machine Publisher.

[50] Mittal, S., Khan, M. A., Romero, D. \& Wuest, T. (2019). Smart manufacturing: Characteristics, technologies, and enabling factors. Proceedings of the Institution of Mechanical Engineers, Part B, Journal of Engineering Manufacture, 233(5), 1342-1361.

[51] Cavalieri, S., \& Cimini, C. (2020). From mass production to smart manufacturing: An evolutionary trajectory and an agenda for future research. International Coalition of Intelligent Manufacturing (ICIM), 151-168.

[52] Elkhova O.I., Kudryashev A. F. (2017). The creative ability of artificial intelligence. Creativity Studies (2), doi:10.3846/23450479.2017.1348396.

[53] Au, S., Berniker, M., Erdogan, A., et al. (2008). Powered ankle-foot prosthesis to assist level-ground and stair-descent gaits. Neural Networks the Official Journal of the International Neural Network Society, 21(4), 654-666.

[54] Mortl, A., Lawitzky, M., Kucukyilmaz, A., et al. (2012). The role of roles: Physical cooperation between humans and robots. The International Journal of Robotics Research,31(13):1656-1674.

[55] Vancza, J., Kemeny, Z., Wang, L., \& Wang, X. V. (2020). Human-robot collaboration in manufacturing: A multi-agent view. International Coalition of Intelligent Manufacturing (ICIM), 190-207.

[56] Daugherty, P. R., \& Wilson, H. J. (2018). Human + Machine: reimagining work in the age of AI. Boston: Harvard Business Review Press, 1-264.

[57] Choudhury, P., Starr, E., Agarwal, R. (2020). Machine learning and human capital complementarities: experimental evidence on bias mitigation. Strategic Management Journal, 41(8):1381-1411.

[58] Guo, L. (2020). Feedback and uncertainty: some basic problems and results. Annual Reviews in Control, 49:27-36.

[59] Araujo Vanessa, S., Rezende Thiago, S., Guimaraes Augusto, J., et al. (2019). A hybrid approach of intelligent systems to help predict absenteeism at work in companies. SN Applied Sciences (6), doi:10.1007/S42452-019-0536-Y. 